\title{
Corrections
}

\section{Correction: Murase et al., Loss of Signal Transducer and Activator of Transcription 3 (STAT3) Signaling during Elevated Activity Causes Vulnerability in Hippocampal Neurons}

In the article "Loss of Signal Transducer and Activator of Transcription 3 (STAT3) Signaling during Elevated Activity Causes Vulnerability In Hippocampal Neurons" by Sachiko Murase, Eunyoung Kim, Lin Lin, Dax A. Hoffman, and Ronald D. McKay, which appeared on pages 15511-15520 of the October 31, 2012 issue, gel images from Figure 3A were inadvertently duplicated in Figure 3B. The revised Figure 3B published here shows correct images. This correction does not affect the results or their interpretation. We also wish to point out that three gel images for $\beta$-actin in Figure 2 of this article also appear in Figure $3 \mathrm{~A}$ because they are from the same experiment.

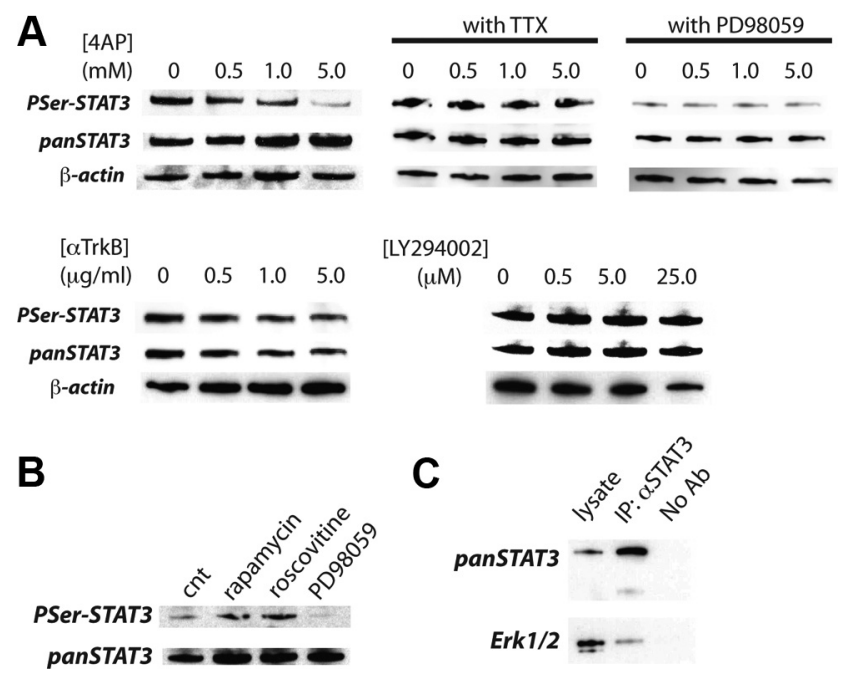

Figure $3 B$.

DOI: $10.1523 / J N E U R O S C I .0262-13.2013$

\section{Correction: Cai et al., A Proactive Mechanism for Selective Suppression of Response Tendencies}

In the article "A Proactive Mechanism for Selective Suppression of Response Tendencies" by Weidong Cai, Caitlin L. Oldenkamp, and Adam R. Aron, which appeared on pages 5965-5969 of the April 20, 2011 issue, the authors incorrectly acknowledged the National Science Foundation for supporting this work, which was solely supported by the Alfred P Sloan Foundation and NIH NIDA Grant DA026452.

DOI: 10.1523/JNEUROSCI.0355-13.2013 